\title{
Adaptive Optical System for Photothermal Microscopy
}

Luis Emiliano $\mathrm{Jan}^{1 *}$, Facundo Zaldivar Escola ${ }^{1,2}$, Nélida Mingolo ${ }^{1}$

${ }^{1 .}$ Laboratorio de Haces Dirigidos, Departamento de Física, (FIUBA), Buenos Aires, Argentina.

2. Consejo Nacional de Investigaciones Científicas y Técnicas (CONICET), Buenos Aires, Argentina.

* Corresponding author: emilianojan@gmail.com

With the advent of the Industry 4.0 and the custom-made manufacturing, new problems have been arisen due to the lack of a statistical set of samples for characterization. This leads to the need of using nondestructive techniques with the capability of adapting to the sample shape. Photothermal techniques are highly effective for this purpose, since it can obtain thermal properties of many materials with local resolution. Particularly, thermal diffusivity allows exposing a high contrast between the phases of a material and, through a microscopy of this property, it is possible to obtain information of the presence of clusters, pores, homogeneity, etc.

The Photothermal Microscopy technique [1], developed by the group, allows the measurement of thermal diffusivity with great precision and its capabilities for the study of metals, ceramics, glass and polymers have been shown [2-4]. It is based on the local measurement of surface curvature, induced by the heating of a modulated pump beam, using a CW probe laser. Both lasers emerge from the same optical fiber and the detected signal corresponds to the reinjection, in the same fiber, of the portion of the probe beam reflected on the curved surface.

The adaptability of the technique to the surface to be measured is based on the development of a novel portable telescope (see Fig. 1), based on DVD lenses. These lenses are coupled to electromagnetic positioners that allow its orientation in order to maintain the focus of the pump and probe beams on the surface and ensuring the maximum reinjection in the fiber, since these parameters define the precision of the technique.

The development of the Adaptative Optical System includes from the design of the optomechanical parts and their electronic controllers, to the construction of algorithms for the automation of all stages and the realization of linear sweeps.

The final lateral resolution of the system is determined by the pump beam waist, being $1 \mu \mathrm{m}$. The measurement duration per point is 30 seconds and the maximum length that can be measured without human intervention is $600 \mu \mathrm{m}$ over the sample plane and a $1.1 \mathrm{~mm}$ in the perpendicular direction.

Thermal diffusivity measurements are presented with accuracy greater than $10 \%$ on samples of PMMA and BK7.

As an example Fig. 2 shows a histogram with the distribution of the measured thermal diffusivity for BK7. The mean value observed was $0.0039 \pm 0.0002 \mathrm{~cm}^{2} / \mathrm{s}$ with a repeatability error in the measurement of $6 \%$. 
References:

[1] N. Mingolo, O.E. Martínez, J. Appl. Phys. 111(12) (2012) p. 123526.

[2] F. Zaldivar Escola et al., Procedia Mater. Sci. 8 (2015), p. 665.

[3] F. Zaldivar Escola , R. Kempf, N.Mingolo, O.E. Martínez, Int. J. Thermophys. 37(20) (2016), p. 1

[4] F. Zaldivar Escola, O.E. Martínez, N. Mingolo, Rev. Mat. 23(2) (2018). p. 1.

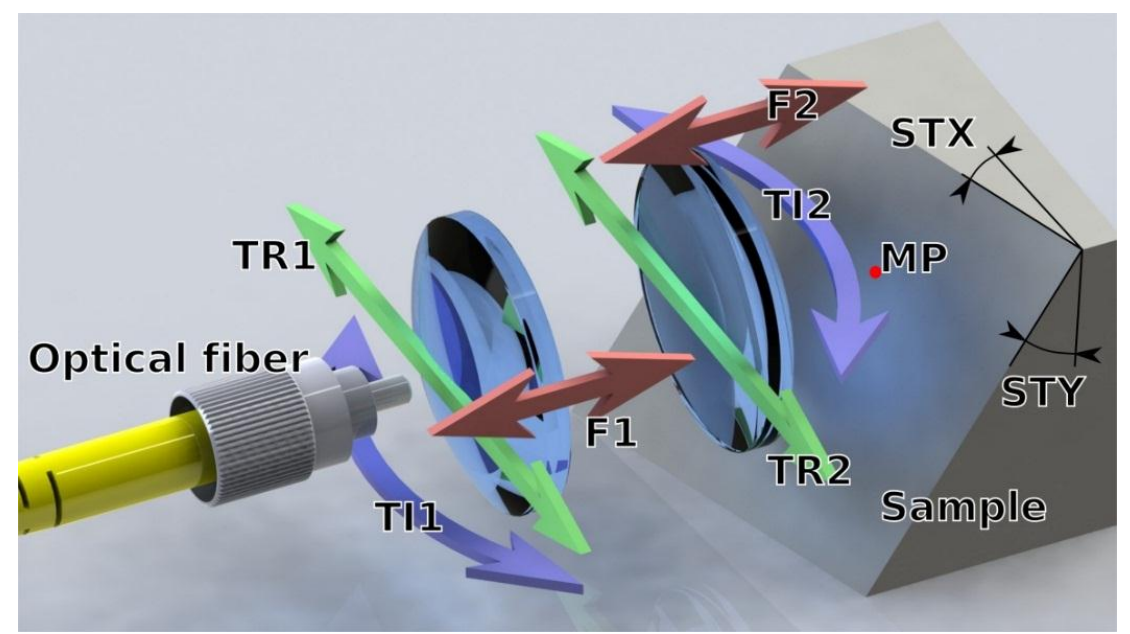

Figure 1. Scheme of the Adaptative Optical System. The pump and probe beams emerge from the Fiber Optic. The lens system allows focusing on the surface of the sample and reinjecting the reflection of the probe beam. STX and STY: tilt angles of the surface. MP: measurement point. The arrows indicate the controllable movements of the DVD lenses. F1 and F2: Focus. TR1 and TR2: Tracking. TI1 and TI2: tilt.

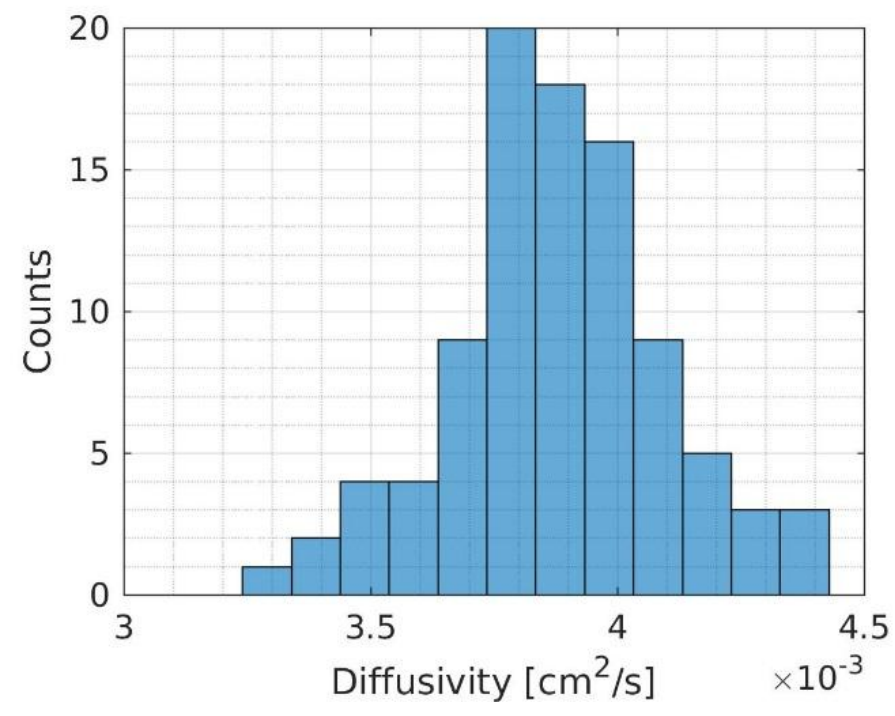

Figure 2. Histogram of diffusivity for a homogeneous sample of BK7. Mean value: $0.0039 \pm 0.0002 \mathrm{~cm}^{2} / \mathrm{s}$. 